\title{
Knockdown of miR-222 inhibits inflammation and the apoptosis of LPS-stimulated human intervertebral disc nucleus pulposus cells
}

\author{
YANG ZHANG, JIUJIE YANG, XIAOQING ZHOU, NAN WANG, ZHI LI, \\ YUBO ZHOU, JIANZHOU FENG, DEWEI SHEN and WEI ZHAO \\ Spine Division, Department of Orthopedics, Central Hospital Affiliated to Shenyang Medical College, \\ Shenyang, Liaoning 110024, P.R. China
}

Received February 25, 2019; Accepted June 14, 2019

DOI: $10.3892 /$ ijmm.2019.4314

\begin{abstract}
It has been demonstrated that miR-222 is upregulated in human intervertebral disc (IVD) degeneration tissues; however, the underlying mechanisms remain unclear. In this study, we aimed to elucidate the mechanisms of action of miR-222 in IVD tissues. Nucleus pulposus (NP) cells were treated with lipopolysaccharide (LPS) to simulate IVD degeneration. The expression level of miR-222 was detected by reverse transcription-quantitative polymerase chain reaction (RT-qPCR) in cells and tissues. Cell apoptosis was analyzed by flow cytometry. Additionally, western blot analysis was used to determine the levels of Toll-like receptor 4 (TLR4), $\mathrm{I} \kappa \beta$-alpha $(\mathrm{I} \kappa \mathrm{B} \alpha)$ and $\mathrm{p} 65$. Interleukin (IL)-1 $\beta$, tumor necrosis factor- $\alpha$ (TNF- $\alpha$ ) and IL-6 protein expression levels were determined by enzyme-linked immunosorbent assay (ELISA). The target gene of miR-222 was determined by TargetScan7.2 and dual luciferase reporter gene analysis. Western blot analysis and RT-qPCR were used to determine the mRNA and protein levels of tissue inhibitor of metalloproteinase 3 (TIMP3). The mRNA expression level of miR-222 was found to be increased in IVD tissues and in LPS-stimulated cells, and its expression was positively associated with the clinical MRI grade. In vitro, apoptosis was promoted/inhibited by miR-222 mimics/inhibitors. Transfection with miR-222 mimics/inhibitors significantly increased/decreased the production of TNF- $\alpha$, IL-1 $\beta$ and IL- 6 and suppressed/enhanced collagen II and aggrecan expression. The protein levels of TLR4, p-I $\mathrm{B} \alpha$ and $\mathrm{p}$-p65 were upregulated/downregulated by transfection with the mimics/inhibitors. In addition, it was demonstrated that TIMP3 was a direct target gene of miR-222,
\end{abstract}

Correspondence to: Dr Wei Zhao, Spine Division, Department of Orthopedics, Central Hospital Affiliated to Shenyang Medical College, 5 Nanqi West Road, Tiexi, Shenyang, Liaoning 110024, P.R. China

E-mail: weiz_zhaowi@163.com

Key words: human intervertebral disc degeneration, miR-222, tissue inhibitor of metalloproteinase 3, Toll-like receptors 4/nuclear factor $\kappa \mathrm{B}$ and was negatively regulated by miR-222 in NP cells. The silencing of TIMP3 reversed the inhibitory effects of miR-222 inhibitor on cell apoptosis, which was induced by LPS. Thus, on the whole, the findings of this study demonstrate that miR-222 functions as a promoter of IVD development, partly via the regulation of TIMP3.

\section{Introduction}

Intervertebral disc (IVD) degeneration is a typical and frequently-occurring disease caused by the degeneration of the IVD, and includes cervical spondylosis, disc herniation and lumbar instability (1-3). At present, scholars agree that IVD degeneration occurs under a variety of physiological and pathological conditions and is affected by a number of factors, such as heredity, cellular senescence, mechanical load, increased degradative enzymes, increased levels of inflammatory factors and apoptosis (4-6). However, the exact mechanisms responsible for IVD degeneration remain unclear.

The IVD is composed of an outer collagen-rich annulus fibrosus and a nucleus pulposus (NP) with a gel-like structure (7). IVD cells, particularly NP cells, can produce collagen II, aggrecan and other components to maintain the integrity of the IVD (8). The reduction in the number of NP cells and the loss of the extracellular matrix are central features of IVD degeneration (9). Another previous study also demonstrated that NP cells are indispensable for maintaining intradiscal balance (10).

It has been widely accepted that the signaling molecules of Toll-like receptor 4 (TLR4) act through intracellular and extracellular pathways to activate the nuclear factor $\kappa \mathrm{B}$ $(\mathrm{NF}-\kappa \mathrm{B})$ signaling pathway to mediate systemic inflammatory responses (11). NF- $\kappa \mathrm{B}$ is located in the cytoplasm under physiological conditions, and its p65 subunit binds to IkB monomer to form a NF- $\kappa \mathrm{B}$ complex, which is in an inactive state, and thus it cannot enter the nucleus to play a regulatory role (12). When the body is stimulated by an external stimulating factor, $\mathrm{IkB}$ is phosphorylated and dissociated from the $\mathrm{NF}-\kappa \mathrm{B}$ dimer. $\mathrm{NF}-\kappa \mathrm{B}$ activates and shifts into the nucleus, binds to specific sites on the DNA strand, and initiates gene transcription to affect protein expression (13), and plays an important role in regulating the body's inflammatory response.

There is increasing evidence to indicate that the mechanisms of apoptosis are triggered by a newly defined small non-coding 
RNA that triggers translational inhibition or RNA degradation to control gene expression by binding to the 3'-UTR of the target gene (14-16). These microRNAs (miRNAs or miRs) act as key factors in regulating various biological processes including proliferation, differentiation, apoptosis, organ development, and inflammatory diseases (17). However, the role of miRNAs in human IVD degeneration have not been reported in detail. As a multifunctional miRNA, miR-222 is expressed in different tissues and is associated with the development of various diseases (18). It has been found that miR-222 is upregulate in clinical patients with IVD degeneration tissue (19), suggesting that miR-222 may be closely related to the process of IVD degeneration. Tissue inhibitor of metalloproteinase 3 (TIMP3) is a member of the metalloproteinase tissue inhibitor family and is an endogenous inhibitor of aggrecanase, which exerts a strong inhibitory effect on aggrecanase activity (20). Previous studies have found that miR-222 can directly target TIMP3 (21-23). Moreover, studies have demonstrated that increasing the expression of TIMP3 inhibits the degeneration of the IVD $(24,25)$.

In this study, we detected the level of miR-222, as well as the expression of TIMP3 in IVD tissues or/and lipopolysaccharide (LPS)-treated NP cells. With in vitro experiments, we further determined whether miR-222 targets TIMP3 directly in NP cells, and we examined the effects of miR-222 on inflammation and on the apoptosis of LPS-treated NP cells, as well as its association with the TLR4/NF- $\mathrm{B}$ signaling pathway.

\section{Materials and methods}

Tissue samples. This study was approved by the Central Hospital Affiliated to Shenyang Medical College Ethics Committee. From January, 2013 to January, 2015, 22 intervertebral disc specimens were collected from the Central Hospital Affiliated to Shenyang Medical College. The 22 IVD patients underwent intervertebral disc excision and spinal fusion surgery. In addition, 9 normal tissues were collected from patients who underwent traumatic lumbar fracture. Written informed consent was obtained from all patients that underwent intervertebral disc excision and spinal fusion surgery, as well as the 9 patients that underwent traumatic lumbar fracture. All specimens were kept anonymous in accordance with ethics and the relevant research laws. All tissue samples were re-evaluated and classified according to the MRI grade and immediately frozen in liquid nitrogen for RNA extraction. Clinical information such as age, sex, body mass index and degeneration level were also collected during follow-up. Clinical follow-up was available to all patients. At the end of the follow-up (5 years), 22 patients remained alive.

Reagents. miR-222 mimic (5'-AGCUACAUCUGGCUACUG GGU-3'), inhibitor (5'-AGCUACAUUGUCUGCUGGGUU UC-3') and mock (5'-UCUACUCUUUCUAGGAGGUUG UGA-3'), which was the negative control used for the transfection of miR-222 mimics and inhibitors, were obtained from GenePharma. TIMP3-siRNA (cat. no. AM16708) and TIMP3-siNC (cat. no. AM4611) were obtained from Ambion (Thermo Fisher Scientific). LPS was purchased from Sigma-Aldrich (cat. no. L2630).
Cells and cell culture. Human nucleus pulposus (NP, cat. no. 4800) cells were obtained from ScienCell Research Laboratories and cultured in Dulbecco's modified Eagle's medium (DMEM, Gibco; Thermo Fisher Scientific) containing $10 \%$ fetal bovine serum (FBS, Gibco; Thermo Fisher Scientific) and antibiotics (1\% penicillin/streptomycin, BBI Life Sciences) at $37^{\circ} \mathrm{C}$ in a humidified atmosphere of $5 \% \mathrm{CO}_{2}$.

Cell treatment. miR-222 mimic, inhibitor and TIMP3-siRNA and the respective controls were used to transiently transfect the NP cells $\left(5 \times 10^{4}\right.$ cells/well $)$ in a 6 -well plate using Lipofectamine 3000 (Thermo Fisher Scientific). At $24 \mathrm{~h}$ following transfection, the cells were stimulated with $1 \mu \mathrm{g} / \mathrm{ml}$ LPS in serum-free medium (DMEM) for $24 \mathrm{~h}$ at $37^{\circ} \mathrm{C}$ under $5 \% \mathrm{CO}_{2}$. The cells were then harvested for subsequent experimentation.

Analysis of cell apoptosis. Annexin V-FITC- propidium iodide (PI) apoptosis detection reagent (BD Biosciences) was used to determine cell apoptosis according to the manufacturer's instructions. Briefly, the cells were washed with PBS 3 times, digested with trypsin $(1 \mathrm{ml})$ and follow resuspended in $1 \mathrm{X}$ Annexin binding buffer at $1 \times 10^{5}$ cells $/ 100 \mu \mathrm{l}$. At room temperature, the cells were collected and stained with Annexin V-FITC and PI for $15 \mathrm{~min}$ and counted by flow cytometry (version 10.0, FlowJo, FACS Calibur ${ }^{\mathrm{TM}}$, BD Biosciences). Cells in the lower left quadrant represent living cells, those in the left upper quadrant represent mechanically damaged or necrotic cells, those in the upper right quadrant represent advanced apoptotic cells and those in the lower right quadrant represent early apoptotic cells.

$R N A$ isolation and reverse transcription-quantitative PCR $(R T-q P C R)$. Total RNA was extracted from the IVD tissues and NP cells using TRIzol reagent (Invitrogen; Thermo Fisher Scientific). Small RNA was isolated from the total RNA using a miRcute miRNA isolation kit (cat. no. DP501, Tiangen Biotech Co.). For mRNA, $1 \mu \mathrm{g}$ of RNA was used to reverse transcribe the RNA into cDNA using the reverse transcription cDNA kit (Thermo Fisher Scientific). SYBR-Green PCR Master Mix (Roche) was used to conduct the qPCR experiments using the Opticon RT-PCR Detection System (ABI 7500, Life technologies). For miRNA, a miRcute plus miRNA first-strand cDNA Synthesis kit (cat. no. KR201-02, Tiangen Biotech) was used to synthesize the cDNA. A miRcute miRNA qPCR Detection kit (cat. no. FP401, Tiangen Biotech) was used for quantification. The PCR cycle was as follows: Pre-treatment at $95^{\circ} \mathrm{C}$ for $10 \mathrm{~min}$; followed by 40 cycles of $94^{\circ} \mathrm{C}$ for $15 \mathrm{sec}$, at $60^{\circ} \mathrm{C}$ for $1 \mathrm{~min}$ finally at $60^{\circ} \mathrm{C}$ for $1 \mathrm{~min}$ and at $4^{\circ} \mathrm{C}$ for preservation. The expression levels of the genes were analyzed using the $2^{-\Delta \Delta \mathrm{Cq}}$ method (26). U6 and GAPDH expression was respectively used for miRNA and mRNA for normalization. The sequences of the primer used are presented in Table I.

Western blot analysis. Total proteins were isolated from the NP cells using RIPA buffer (Cell Signaling Technology, Inc.). The BCA Protein Assay kit (Pierce) was applied to measure the protein concentration, which was adjusted to a concentration of $6 \mu \mathrm{g} / \mu \mathrm{l}$ using $1 \mathrm{X}$ loading buffer and DEPC water. Electrophoresis was used to separate the samples using a $10 \%$ 
Table I. Sequences of primers used for RT-qPCR.

\begin{tabular}{lll}
\hline Genes & \multicolumn{1}{c}{ Forward } & \multicolumn{1}{c}{ Reverse } \\
\hline Collagen II & CTGGTGATGATGGTGAAG & CCTGGATAACCTCTGTGA \\
Aggrecan & GTGGGACTGAAGTTCTTG & GTTGTCATGGTCTGAAGTT \\
TIMP3 & GTGCAACTTCGTGGAGAGGT & AGCAGGACTTGATCTTGCAGT \\
GAPDH H & GCACCGTCAAGGCTGAGAAC & TGGTGAAGACGCCAGTGGA \\
miR-22 & AGCTACATCTGGCTACTGG & GTATCCAGTGCAGGGTCC \\
U6 & CTCGCTTCGGCAGCACA & TGGTGTCGTGGAGTCG \\
\hline
\end{tabular}

SDS-PAGE gel followed by transfer onto a polyvinylidene fluoride membrane (PVDF, Millipore). After blocking in 5\% non-fat milk in PBST [0.1\% Tween-20 in phosphate-buffered saline (PBS)] for $1 \mathrm{~h}$, the membrane was incubated with the primary antibody overnight at $4^{\circ} \mathrm{C}$, and subsequently incubated with secondary antibody [horseradish peroxidase (HRP)-conjugated goat anti-mouse/rabbit IgG, 1:2,000; sc-516102/sc-2357; Santa Cruz Biotechnology, Inc.] at room temperature for $2 \mathrm{~h}$. Developer (EZ-ECL kit; Biological Industries BI) was used for development, and the gray value of the strips were analyzed and quantified using image J software (version 5.0; Bio-Rad). The antibodies utilized included anti-GAPDH (mouse; 1:1,000; LS-B1625; LifeSpan BioSciences, Inc.), anti-TLR4 (rabbit; 1:500; ab13556; Abcam),

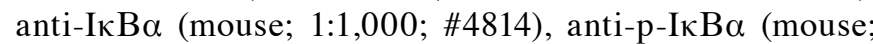
1:1,000; \#9246), anti-p65 (rabbit; 1:1,000; \#8242), anti-p-p65 (rabbit; 1:1,000; \#3039) and anti-TIMP3 (rabbit; 1:1,000; \#5673) (all from Cell Signaling Technology, Inc.).

Enzyme-linked immunosorbent assay (ELISA). The serum levels of TNF- $\alpha$, IL- $1 \beta$ and IL- 6 were measured using ELISA kits (eBioscience) according to the manufacturer's instructions. All standards and samples were measured using a microplate reader (SpectraMax M5, Molecular Devices) at a wavelength of $450 \mathrm{~nm}$, a standard curve was prepared using computer software, and the corresponding sample concentration was calculated based on the absorbance value.

Bioinformatics prediction. The potential target genes of miR-222 were predicted using TargetScan7.2 online software (http://www.targetscan.org/vert_72/), according to the manufacturer's instructions. 'miR-222' was inserted and 'human' was selected. The putative target genes of miR-222 were scanned.

Binding ability of TIMP3 to miR-222. 293 cells (BeNa Culture Collection) were transfected with $100 \mathrm{~nm}$ TIMP3-3'-UTR plasmid [the TIMP3 mutant (MT) and wild-type (WT)] (GeneChem), as well as with or without $100 \mathrm{~nm}$ miR-222. Subsequently, the luciferase reporter assay system (Promega Corp.) was used to measure luciferase activity in Lmax II luminescence meter (Molecular Devices, LLC) for $48 \mathrm{~h}$ following transfection. Renilla luciferase activity was defined as the standardization of Firefly luciferase activity.

Statistical analysis. Statistical analysis was carried out using Prism 6 software (GraphPad Software, Inc.). Statistically significant differences between groups were determined using one-way analysis of variance (ANOVA), followed by Bonferroni's post hoc test. The Chi-square test was used for the discontinuous variables shown in Table II. The results are presented as the means \pm standard deviation (SD) and statistically significant differences are indicated by $\mathrm{P}<0.05$.

\section{Results}

The expression level of miR-222 is associated with the clinicopathological characteristics of IVD degeneration. The association between the expression of miR-222 and the clinicopathologic characteristics of the patients with IVD were evaluated (Table II). A high miR-222 expression was associated with a high MRI grade $(\mathrm{P}=0.029)$. However, the miR-222 expression level was not associated with age, sex, body mass index or the degeneration level status.

miR-222 inhibitor decreases LPS-induced NP cell apoptosis. We determined the level of miR-222 in IVD tissues and an IVD cell model was generated using NP cells treated with LPS. The results revealed that miR-222 expression was significantly increased in IVD tissues (Fig. 1A) compared with the normal controls. Its expression was also increased in a dose-dependent manner in the LPS-treated NP cells (Fig. 1B). Following the referral to relevant literature and the experimental results, the concentration of $1 \mu \mathrm{g} / \mathrm{ml}$ LPS was selected for use in later experiments (27). To further examine the effect of miR-222 on LPS-induced NP cell apoptosis, miR-222 mock, mimics and inhibitor were transfected into the NP cells, and a high transfection efficiency was observed (Fig. 1C). The results of the flow cytometric analysis of apoptosis demonstrated that cell apoptosis was decreased in the miR-222 inhibitor-transfected NP cells compared with the LPS-treated cells, while miR-222 mimics exerted the opposite effect (Fig. 1D).

miR-222 inhibitor decreases pro-inflammatory cytokine levels and enhances collagen II and aggrecan expression in LPS-stimulated NP cells. Subsequently, we examined the expression levels of TNF- $\alpha$, IL- $1 \beta$ and IL- 6 in NP cells stimulated with LPS for $24 \mathrm{~h}$ by ELISA. As expected, we found that compared with the cells stimulated with LPS alone, transfection with miR-222 inhibitor led to a marked decrease in the levels of TNF- $\alpha$, IL-1 $\beta$ and IL- 6 . By contrast, transfection with miR-222 mimics markedly increased the production of TNF- $\alpha$, IL- $1 \beta$ and IL- 6 in the LPS-stimulated 
Table II. Association between miR-222 expression and the clinical characteristics of patients with IVD degeneration.

\begin{tabular}{lrrrr}
\hline & miR-222 expression & & \\
\cline { 2 - 3 } Parameters & Low (\%) & High (\%) & Total & P-value \\
\hline Age (years) & & & & 0.096 \\
$\leq 45$ & $6(27.3)$ & $3(13.6)$ & 9 & \\
$>45$ & $4(18.2)$ & $9(40.9)$ & 13 & \\
Sex & & & & 0.145 \\
Female & $2(9.1)$ & $6(27.3)$ & 8 & \\
Male & $8(36.4)$ & $6(27.3)$ & 14 & \\
Body mass index & & & & 0.937 \\
$\leq 24$ kg/m ${ }^{2}$ & $4(18.2)$ & $5(22.7)$ & 9 & \\
$>24$ kg/m ${ }^{2}$ & $6(27.3)$ & $7(31.8)$ & 13 & \\
Degeneration level & & & & 0.190 \\
L3/4 & $5(22.7)$ & $3(13.6)$ & 8 & \\
L4/5 & $2(9.1)$ & $7(31.8)$ & 9 & \\
L5/S1 & $3(13.6)$ & $2(9.1)$ & 5 & \\
MRI grade & & & & $0.029^{a}$ \\
G (I/II) & $8(36.4)$ & $4(18.2)$ & 12 & \\
G (IV/V) & $2(9.1)$ & $8(36.4)$ & 10 & \\
\hline
\end{tabular}

${ }^{a}$ Indicates statistical significance $(\mathrm{P}<0.05)$. IVD, intervertebral disc.

cells (Fig. 2A-C). The effects of miR-222 on collagen II and aggrecan expression in the NP cells were also examined by RT-qPCR, and the data indicated that the expression levels of collagen II and aggrecan were lower in the LPS group than those in the control group. Compared with the cells stimulated with LPS only, transfection with miR-222 mimics significantly inhibited collagen II and aggrecan expression in the NP cells, while transfection with miR-222 inhibitor markedly enhanced the collagen II and aggrecan levels (Fig. 2D and E).

The levels of TLR4, $p$-I $\kappa B \alpha$ and $p-p 65$ are suppressed by $m i R-222$ inhibitor. In view of the significant role played by the TLR4/ NF- $\kappa B$ signaling pathway in the progression of IVD, we assessed the expression of TLR4 and the phosphorylation levels of I $\mathrm{B} \alpha$ and $\mathrm{p} 65$ in human NP cells stimulated with LPS by western blot analysis. The results demonstrated that the levels of TLR4, p-IкB $\alpha$ and p-p65 were significantly higher in the LPS-treated NP cells than those in the control NP cells. Transfection with miR-222 mimics significantly upregulated the TLR4, $\mathrm{p}$-I $\kappa \mathrm{B} \alpha$ and p-p65 expression levels in NP cells, while transfection with miR-222 inhibitor markedly downregulated the TLR4, p-IкB $\alpha$ and p-p65 levels, when compared with the cells stimulated with LPS only (Fig. 3A). The relative protein levels of TLR4, p-IKB $\alpha$ and p-p65 in NP cells are presented in Fig. 3B-D).

miR-222 increases the expression of TIMP3 by directly binding to TIMP3 3'-UTR. To examine the functions of miR-222 in NP cells, we first predicted the potential targets of miR-222 using TargetScan 7.2 and found that the 3'-UTR of TIMP3 had a binding site to miR-222 (Fig. 4A), and dual luciferase reporter assay was applied to confirm our prediction (Fig. 4B). The luciferase activities significantly decreased after miR-222-3p mimic and TIMP3 3'-UTR-wt were co-transfected into the 293 cells. However, the luciferase activities in the cells co-transfected with TIMP3 3'-UTR-mut and miR-222-3p mimic remained stable. Furthermore, the expression level of TIMP3 was markedly downregulated in the LPS-treated NP cells in comparison to the untreated cells, and the expression level of miR-222 was negatively associated with TIMP3 expression at the mRNA (Fig. 4C) and protein level (Fig. 4D and E).

Inhibitory effects of miR-222 inhibitor on cell apoptosis are reversed by transfection with TIMP3-siRNA. As the overexpression miR-222 decreased the TIMP3 levels in the LPS-stimulated NP cells, we then wished to determine whether TIMP3 is involved in the apoptosis of LPS-stimulated NP cells. The NC-siRNA and TIMP3-siRNA plasmids were transfected into the NP cells with/without miR-222 inhibitor and cell apoptosis was examined. The successful knockdown of TIMP3 was verified by RT-qPCR (Fig. 5A) and western blot analysis (Fig. 5B and C). We further analyzed the apoptosis of LPS-stimulated NP cells, and the results revealed that the decrease in the expression of TIMP3 effectively attenuated the inhibitory effects on the apoptosis of LPS-treated NP cells induced by miR-222 inhibition (Fig. 5D).

\section{Discussion}

miRNA-222 has been shown to be encoded in the progression and development of cancer, thereby regulating the proliferation, migration and apoptosis of cancer cells (28-30). miR-221, the same cluster of miR-222, also plays a significant role in the progression of cancer $(31,32)$. Penolazzi et al reported that miR-221 may play a significant role in the etiology of IVD degeneration and that its downregulation may play a pivotal role in the preservation of disc homeostasis and in supporting the endogenous repair process (33). However, the regulation of miR-222 in IVD degeneration has not yet been reported, at least to the best of our knowledge. A recent study stated that miR-222 was highly expressed in IVD degeneration and identified this miRNA as the key to IDD development (19). The results of this study also confirmed that miR-222 was highly expressed in patients with IVD degeneration. These results suggest that miR-222 plays an important role in IVD degeneration and that the understanding of the underlying mechanisms of the regulation of IVD degeneration by miR-222 may provide a promising strategy for the treatment of IVD degeneration.

IVD degeneration is accompanied by NP cell apoptosis, which is primarily induced by TNF-a, and a major characteristic of IVD degeneration is the reduction of the collagen II and aggrecan content in NP cells $(34,35)$. LPS, an admitted strong promoter of inflammation, can reduce the collagen II and aggrecan content, thus leading to IVD degeneration (36). Thus, in the present study, we used LPS to generate the IVD degeneration cell model using NP cells for the further study of miR-222, and found miR-222 was upregulated in human NP cells stimulated with LPS in a dose-dependent manner. Moreover, the collagen II and aggrecan levels were 

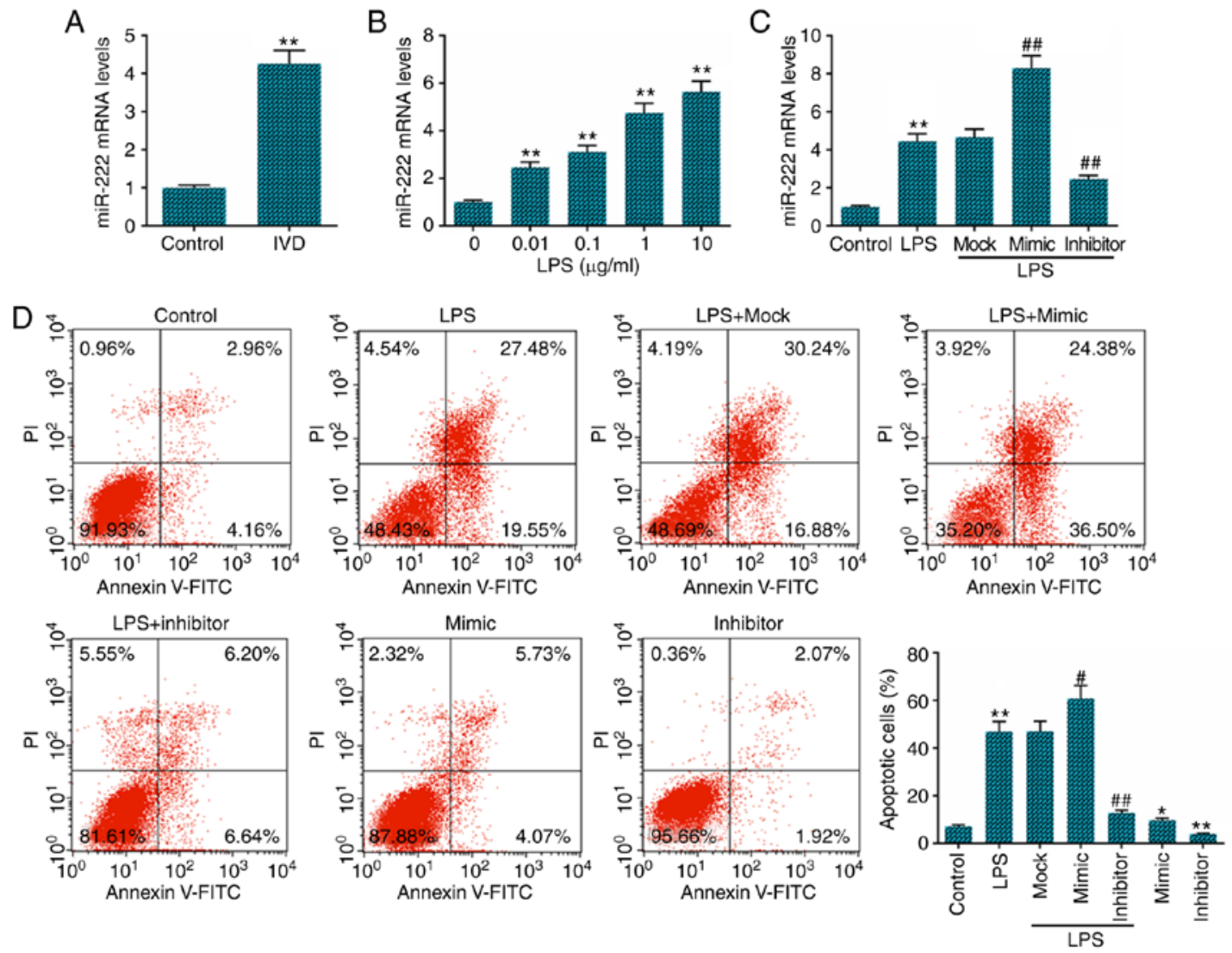

Figure 1. LPS-induced nucleus pulposus cell apoptosis is inhibited by miR-222 inhibitor. (A) miR-222 level in human intervertebral disc tissues was detected by reverse transcription-quantitative polymerase chain reaction. (B) miR-222 level in lipopolysaccharide-stimulated nucleus pulposus cells was detected using reverse transcription-quantitative polymerase chain reaction. (C) Transfection efficiency of miR-222 mock, mimics and inhibitor was determined by reverse transcription-quantitative polymerase chain reaction. (D) Nucleus pulposus cell apoptosis was analyzed by flow cytometry. ${ }^{*} \mathrm{P}<0.05$ and ${ }^{* *} \mathrm{P}<0.01$ vs. control; ${ }^{\#} \mathrm{P}<0.05$ and ${ }^{\# \#} \mathrm{P}<0.01$ vs. cells stimulated with LPS only. LPS, lipopolysaccharide.
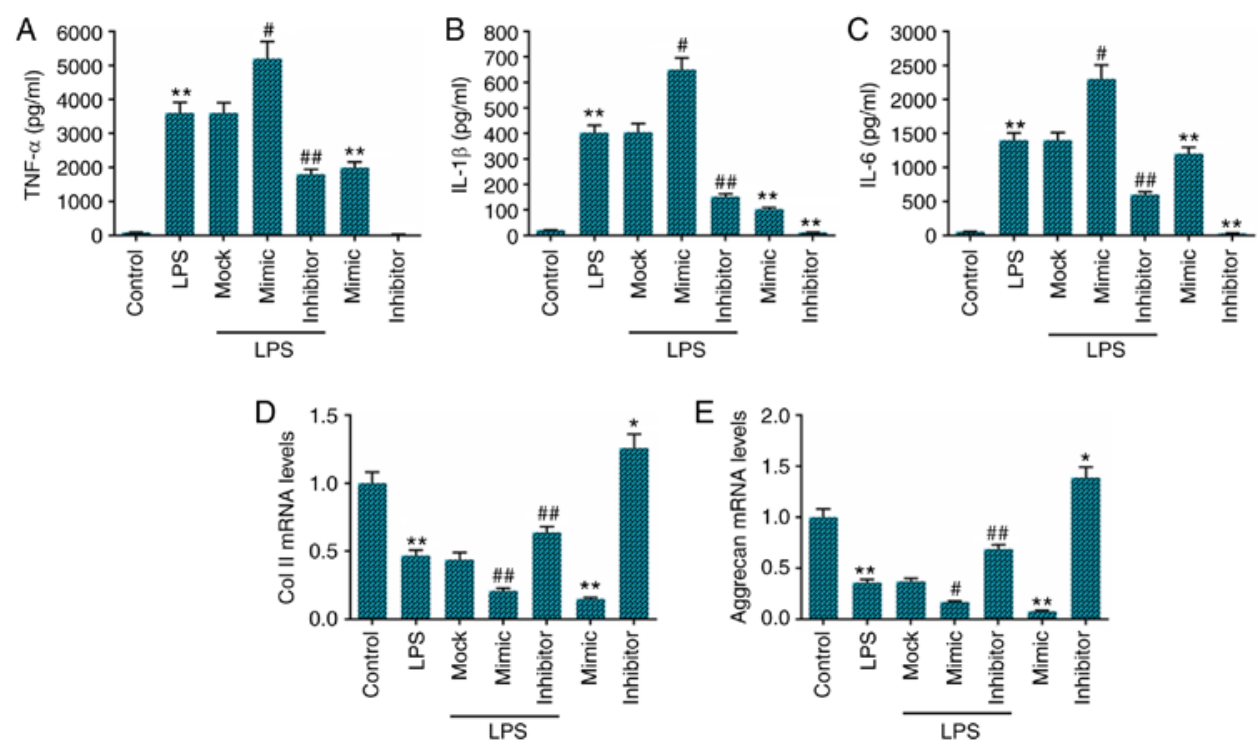

Figure 2. Effects of miR-222 on TNF- $\alpha$, IL-1 $\beta$ and IL-6, collagen II and aggrecan expression levels in LPS-treated nucleus pulposus cells. (A) TNF- $\alpha$, (B) IL-1 $\beta$ and (C) IL-6 expression levels in nucleus pulposus cells treated with LPS were detected by ELISA. The expression levels of (D) collagen II and (E) aggrecan in nucleus pulposus cells treated with LPS were detected using reverse transcription-quantitative polymerase chain reaction ${ }^{*} \mathrm{P}<0.05$ and ${ }^{* * *} \mathrm{P}<0.01$ vs. control, ${ }^{\#} \mathrm{P}<0.05$ and ${ }^{\# \#} \mathrm{P}<0.01$ vs. cells stimulated with LPS only. TNF- $\alpha$, tumor necrosis factor- $\alpha$; IL, interleukin; LPS, lipopolysaccharide.

markedly decreased in the LPS-stimulated NP cells, and these effects were partially reversed by transfection with miR-222 inhibitor, while transfection with miR-222 mimics exerted opposite effects. Those outcomes demonstrate that miR-222 plays a role in the progression of IVD degeneration, at least in LPS-stimulated NP cells. 

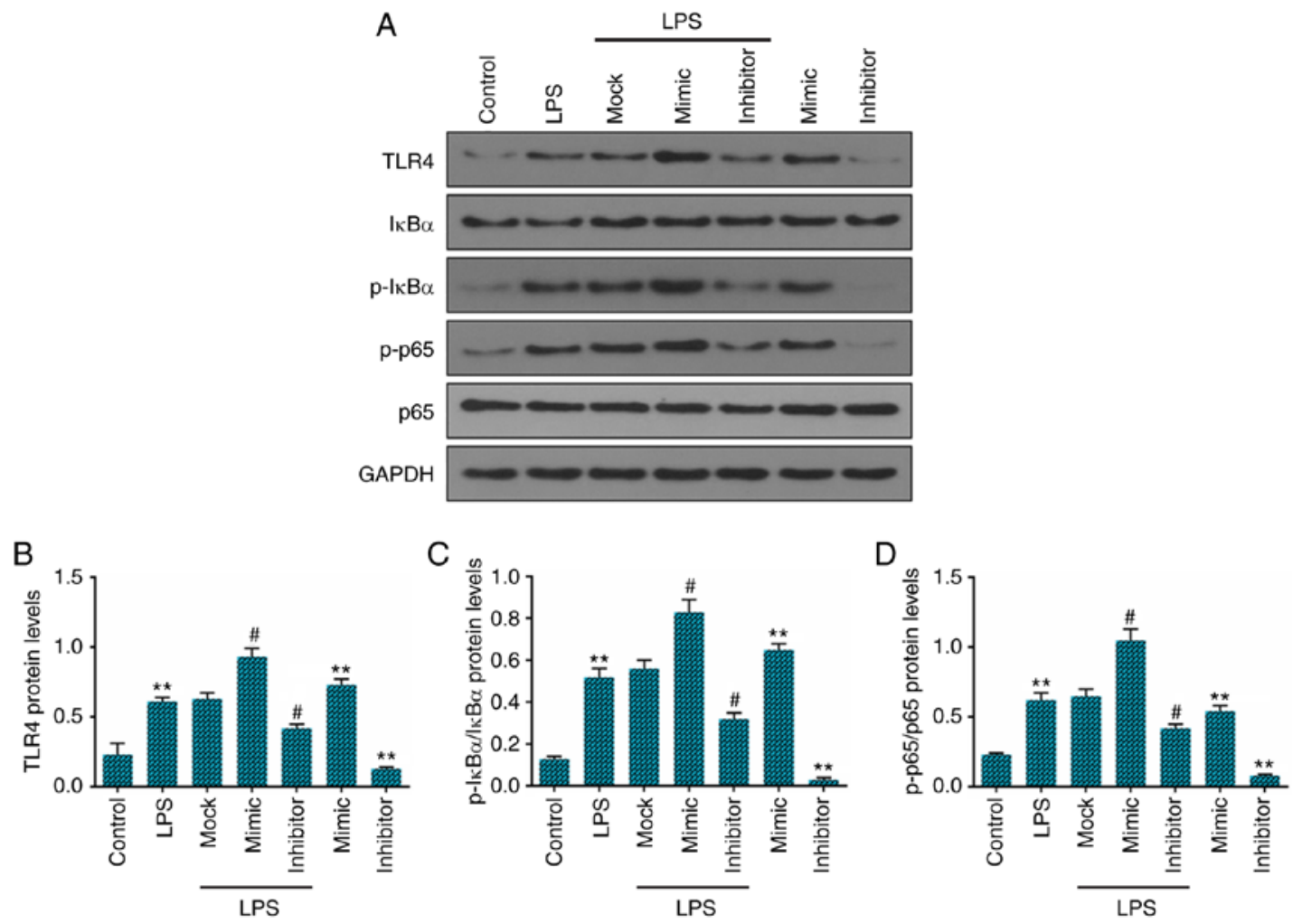

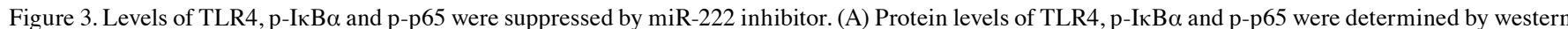
blot analysis. The relative levels of proteins described in (B, C and D) were normalized to ${ }^{* *} \mathrm{P}<0.01$ vs. control; ${ }^{*} \mathrm{P}<0.05$ vs. cells stimulated with LPS only. TLR4, Toll-like receptor 4; LPS, lipopolysaccharide.
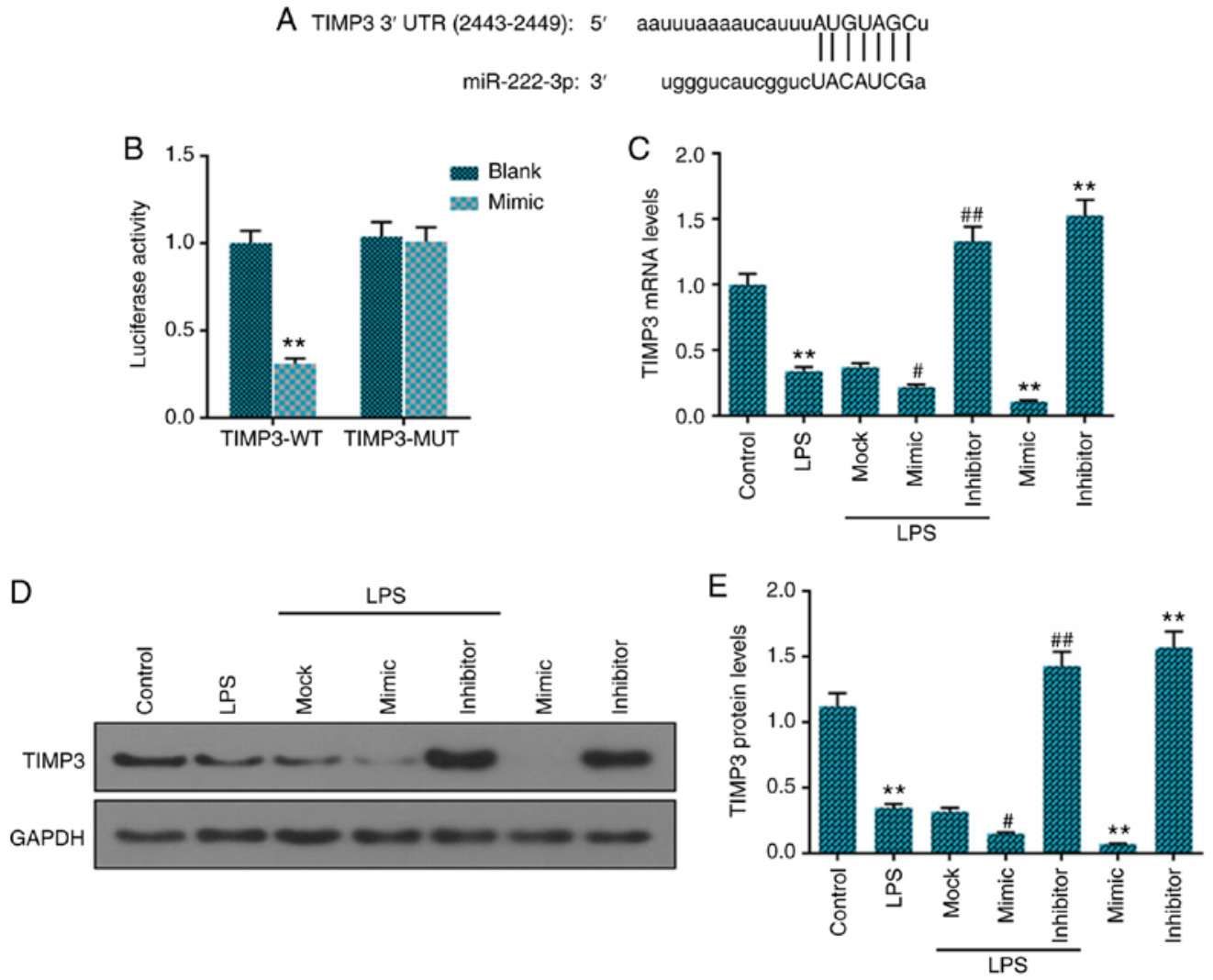

Figure 4. miR-222 targets TIMP3. (A) The 3'-UTR of TIMP3 was predicted as a miR-222 target using TargetScan 7.2. (B) Luciferase activity of a reporter containing a wild-type (WT) TIMP3 3' UTR or a mutant (MUT) TIMP3 3' UTR is shown. (C) The expression of TIMP3 was detected using reverse transcription-quantitative polymerase chain reaction. (D) The protein level of TIMP3 was determined by western blot analysis. The relative level of TIMP3 described in (E) was normalized to ${ }^{* *} \mathrm{P}<0.01$ vs. control; ${ }^{\#} \mathrm{P}<0.05$ and ${ }^{\# \#} \mathrm{P}<0.01$ vs. cells stimulated with LPS only. TIMP3, tissue inhibitor of metalloproteinase 3 ; LPS, lipopolysaccharide. 
A

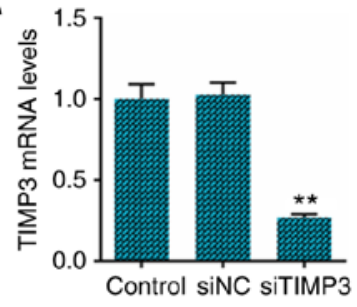

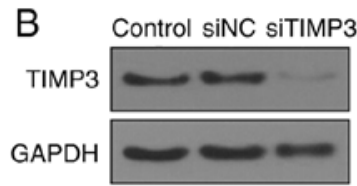

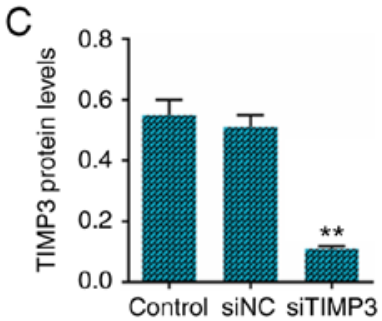

D
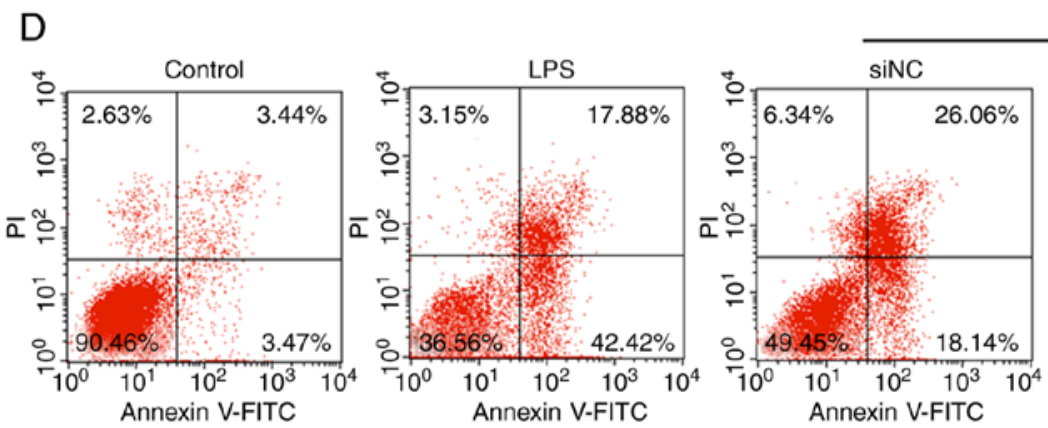

LPS

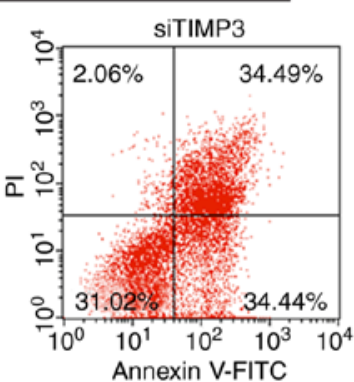

LPS
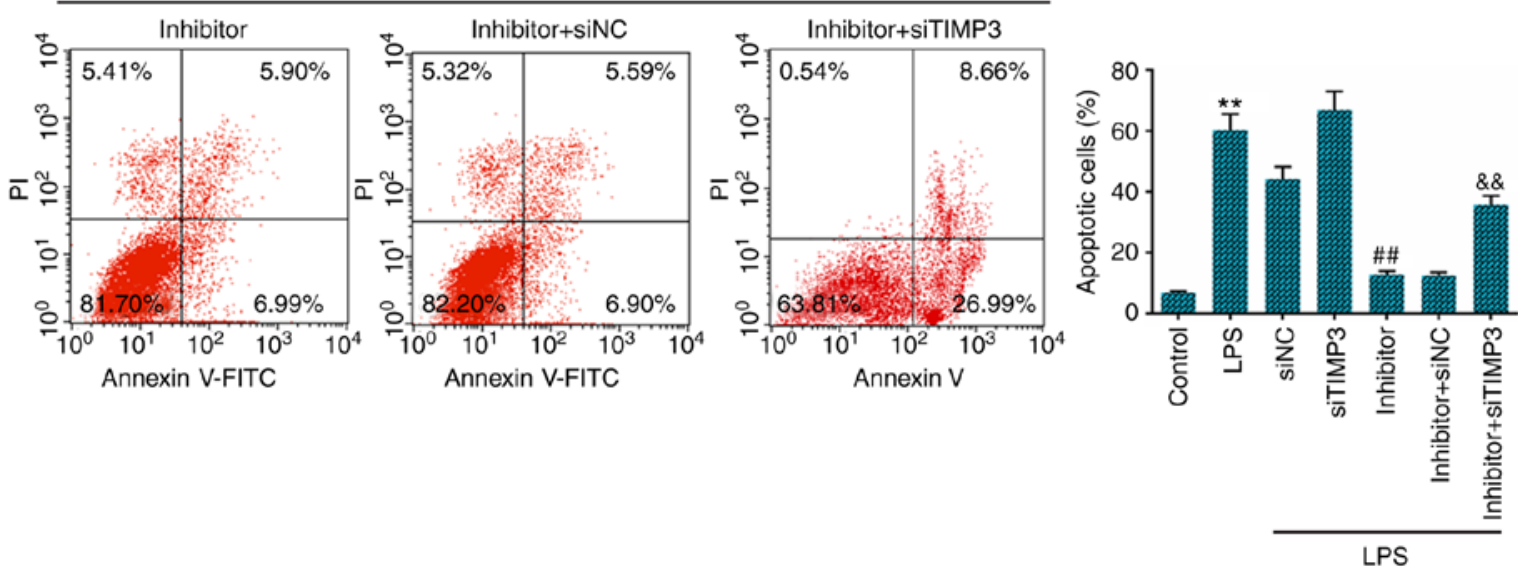

Figure 5. Overexpression of TIMP3 reverses the apoptosis of LPS-stimulated nucleus pulposus cells induced by miR-222 inhibitor. (A) TIMP3 mRNA levels was determined by reverse transcription-quantitative polymerase chain reaction. (B) TIMP3 protein levels were determined by western blot analysis. (C) The relative TIMP3 protein level is presented. (D) Cell apoptosis was analyzed by flow cytometry. ${ }^{* *} \mathrm{P}<0.01 \mathrm{vs}$. control; ${ }^{\# \#} \mathrm{P}<0.01$ vs. cells stimulated with LPS only; ${ }^{\&} \mathrm{P}<0.01$ vs. inhibitor + siNC.

Related studies have demonstrated that disc degeneration is associated with a variety of pro-inflammatory cytokines $(37,38)$. The inhibition of the IL-1 $\beta$-mediated inflammatory response can effectively alleviate IVD degeneration in NP cells (39). TNF and IL play a key role in the development of intervertebral disc degeneration (37). The overexpression of miR-140-5p has been shown to inhibit LPS-induced human IVD inflammation and degeneration by downregulating TLR4 (27). Previous studies have also reported that the inhibition of the NF- $\mathrm{kB}$ signaling pathway can protect against IVD degeneration $(40,41)$. It is well known that the TLR4/NF- $\mathrm{BB}$ signaling pathway mediates inflammation cascades to amplify the inflammatory response $(42,43)$. In this study, the levels of TNF- $\alpha$, IL- $1 \beta$ and IL-6 were markedly lower in the LPS + inhibitor group than that in the LPS-stimulated NP cells, while miR-222 mimics enhanced the production of TNF- $\alpha$, IL-1 $\beta$ and IL- 6 , in comparison to the LPS group. Moreover, significantly decreased expression levels of TLR4, p-I $\kappa \mathrm{B} \alpha$ and p-p65 were observed following transfection with miR-222 inhibitor, while transfection with miR-222 mimics increased the TLR4, $\mathrm{p}$-I $\mathrm{KB} \alpha$ and p-p65 expression levels, when compared with the LPS group. These data suggest that miR-222 may affect the development of IVD degeneration by aggravating the inflammatory response.

Apoptosis, as a physiological process, can be used to remove harmful or severely damaged cells and organelles; however, when this process becomes excessive, it can lead to pathological phenomena (44). The apoptosis of human IVD tissue has been detected and it has been found that a large part of the human IVD during degeneration had undergone programmed cell death (45). Zhang et al found that the injection of the shRNA vector, CHOP shRNA, into the rat IVD inhibited the apoptosis of IVD cells, thereby attenuating the degeneration of the IVD (46). In the present study, NP cells were examined using Annexin V and PI double staining, which can detect the occurrence of apoptosis (47). Compared to the NP cells stimulated with LPS only, apoptosis was inhibited by transfection with miR-222 inhibitor, while transfection with miR-222 mimics promoted cell apoptosis. These result prove that miR-222 plays a key role in IVD degeneration partly by enhancing cell apoptosis. 
miRNAs mostly functions by targeting the 3'-UTR of their target genes (48). It has been found that the increased expression of TIMP3 inhibist the degeneration of the IVD $(24,25)$. Furthermore, TIMP 3 has been reported to be a target of miR-222 $(23,49)$. In the present study, we determined TIMP3 as the target of miR-222 in IVD degeneration, and a significantly increased expression of TIMP3 was observed following transfection with miR-222 inhibitor. However, transfection with miR-222 mimics decreased TIMP3 expression in the LPS-stimulated NP cells. Furthermore, to better understand the role of TIMP3 in IVD, the cells were transfected with TIMP3-siRNA and the results revealed that TIMP3-siRNA attenuated the inhibitory effects on the apoptosis of LPS-treated NP cells induced by miR-222 inhibition. These data demonstrated that miR-222 promoted the progression of IVD degeneration partly by targeting TIMP3.

Although this study demonstrated that miR-222 was highly expressed in IVD degeneration and participated in the process of IVD degeneration, there are several limitations. First, the function of miR-222 downregulation in protecting IVD against degeneration by reducing inflammation and inhibiting apoptosis under LPS stimulation was only supported by in vitro experiments. Additionally, this research has not been confirmed by other studies; thus, further experiments using cells and animals, as well as clinical studies are required to confirm our findings. Other limitations are, for example, the lacking of anti-apoptotic factors induced by NF- $\mathrm{KB}$ signaling. Therefore, we aim to carry out a more comprehensive investigation in the future.

Based on the data described above, in this study, we demonstrated that miR-222 promoted inflammation and apoptosis in IVD degeneration partly by targeting TIMP3 mRNA, and that the knockdown miR-222 reversed these effects in LPS-stimulated NP cells. These results provide further insight into the study of IVD degeneration and may prove to be of regulatory and diagnostic importance in the study of IVD degeneration.

\section{Acknowledgements}

Not applicable.

\section{Funding}

No funding was received.

\section{Availability of data and materials}

The analyzed datasets generated during the study are available from the corresponding author on reasonable request.

\section{Authors' contributions}

WZ and YaZ made substantial contributions to the conception and design of the study. JY and $\mathrm{XZ}$ were involved in data acquisition. NW, ZL and DS were involved in data analysis. YuZ and JF were involved in data interpretation. YaZ and NW were involved in the drafting of the article. WZ and JF critically revised the manuscript for important intellectual content. All authors have read and approved the final manuscript. All the authors agree to be accountable for all aspects of the work in ensuring that questions related to the accuracy or integrity of the work are appropriately investigated and resolved.

\section{Ethics approval and consent to participate}

All procedures performed in studies involving human participants were in accordance with the ethical standards of the institutional and/or national research committee and with the 1964 Helsinki declaration and its later amendments or comparable ethical standards. This study was approved by the Central Hospital Affiliated to Shenyang Medical College Ethics Committee. Written informed consent was obtained from all patients that underwent intervertebral disc excision and spinal fusion surgery, as well as the 9 patients that underwent traumatic lumbar fracture.

\section{Patient consent for publication}

Not applicable.

\section{Competing interests}

The authors declare that they have no competing interests.

\section{References}

1. Taher F, Essig D, Lebl DR, Hughes AP, Sama AA, Cammisa FP and Girardi FP: Lumbar degenerative disc disease: Current and future concepts of diagnosis and management. Adv Orthop 2012: 970752, 2012.

2. Chan SC and Gantenbein-Ritter B: Intervertebral disc regeneration or repair with biomaterials and stem cell therapy-feasible or fiction? Swiss Med Wkly 142: w13598, 2012.

3. Park JB, Lee JK, Park SJ, Kim KW and Riew KD: Mitochondrial involvement in fas-mediated apoptosis of human lumbar disc cells. J Bone Joint Surg Am 87: 1338-1342, 2005.

4. Solovieva S, Lohiniva J, Leino-Arjas P, Raininko R, Luoma K, Ala-Kokko L and Riihimäki H: Intervertebral disc degeneration in relation to the COL9A3 and the IL-1ss gene polymorphisms. Eur Spine J 15: 613-619, 2006.

5. Guehring T, Omlor GW, Lorenz H, Bertram H, Steck E, Richter W, Carstens C and Kroeber M: Stimulation of gene expression and loss of anular architecture caused by experimental disc degeneration-an in vivo animal study. Spine (Phila Pa 1976) 30: 2510-2515, 2005.

6. Stokes IA and Iatridis JC: Mechanical conditions that accelerate intervertebral disc degeneration: Overload versus immobilization. Spine (Phila Pa 1976) 29: 2724-2732, 2004

7. Migliorini F, Rath B, Tingart M, Baroncini A, Quack V and Eschweiler J: Autogenic mesenchymal stem cells for intervertebral disc regeneration. Int Orthop 43: 1027-1036, 2019.

8. Johnson ZI, Shapiro IM and Risbud MV: Extracellular osmolarity regulates matrix homeostasis in the intervertebral disc and articular cartilage: Evolving role of TonEBP. Matrix Biol 40: $10-16,2014$

9. Aguiar DJ, Johnson SL and Oegema TR: Notochordal cells interact with nucleus pulposus cells: Regulation of proteoglycan synthesis. Exp Cell Res 246: 129-137, 1999.

10. Hsieh AH and Twomey JD: Cellular mechanobiology of the intervertebral disc: New directions and approaches. J Biomech 43: 137-145, 2010.

11. McCarty MF: Preclinical studies suggest complex nutraceutical strategies may have potential for preventing and managing sepsis. Altern Ther Health Med 21 (Suppl 2): S56-S67, 2015.

12. Gordon JW, Shaw JA and Kirshenbaum LA: Multiple facets of NF-kB in the heart: To be or not to NF-kB. Circ Res 108: 1122-1132, 2011.

13. Ghosh S, May MJ and Kopp EB: NF-kappa B and Rel proteins: Evolutionarily conserved mediators of immune responses. Annu Rev Immunol 16: 225-260, 1998. 
14. Bartel DP: MicroRNAs: Genomics, biogenesis, mechanism, and function. Cell 116: 281-297, 2004.

15. Jovanovic M and Hengartner MO: miRNAs and apoptosis: RNAs to die for. Oncogene 25: 6176-6187, 2006.

16. Selbach M, Schwanhausser B, Thierfelder N, Fang Z, Khanin R and Rajewsky N: Widespread changes in protein synthesis induced by microRNAs. Nature 455: 58-63, 2008.

17. Ma Y, Yu S, Zhao W, Lu Z and Chen J: miR-27a regulates the growth, colony formation and migration of pancreatic cancer cells by targeting Sprouty2. Cancer Lett 298: 150-158, 2010.

18. Wei T, Ye P, Peng X, Wu LL and Yu GY: Prognostic value of miR-222 in various cancers: A systematic review and meta-analysis. Clin Lab 62: 1387-1395, 2016.

19. Hu P, Feng B, Wang G, Ning B and Jia T: Microarray based analysis of gene regulation by microRNA in intervertebral disc degeneration. Mol Med Rep 12: 4925-4930, 2015.

20. Balkowiec M, Maksym RB and Wlodarski PK: The bimodal role of matrix metalloproteinases and their inhibitors in etiology and pathogenesis of endometriosis (Review). Mol Med Rep 18: 3123-3136, 2018

21. Lu Y, Roy S, Nuovo G, Ramaswamy B, Miller T, Shapiro C, Jacob ST and Majumder S: Anti-microRNA-222 (anti-miR-222) and $-181 \mathrm{~B}$ suppresses growth of tamoxifen-resistant xenografts in mouse by targeting TIMP3 protein and modulating mitogenic signal. J Biol Chem 293: 3588, 2018.

22. Zhang C, Zhang J, Hao J, Shi Z, Wang Y, Han L, Yu S, You Y, Jiang T, Wang J, et al: High level of miR-221/222 confers increased cell invasion and poor prognosis in glioma. J Trans Med 10: 119, 2012

23. Lei Y, Liu Z and Yang W: Negative correlation of cytoplasm TIMP3 with miR-222 indicates a good prognosis for NSCLC. Onco Targets Ther 11: 5551-5557, 2018.

24. Li Y, Li K, Han X, Mao C, Zhang K, Zhao T and Zhao J: The imbalance between TIMP3 and matrix-degrading enzymes plays an important role in intervertebral disc degeneration. Biochem Biophys Res Commun 469: 507-514, 2016.

25. Kwon WK, Moon HJ, Kwon TH, Park YK and Kim JH: The role of Hypoxia in angiogenesis and extracellular matrix regulation of intervertebral disc cells during inflammatory reactions Neurosurgery 81: 867-875, 2017.

26. Livak KJ and Schmittgen TD: Analysis of relative gene expression data using real-time quantitative PCR and the 2(-Delta Delta $\mathrm{C}(\mathrm{T})$ ) method. Methods 25: 402-408, 2001.

27. Zhang Q, Weng Y, Jiang Y, Zhao S, Zhou D and Xu N Overexpression of miR-140-5p inhibits lipopolysaccharide-induced human intervertebral disc inflammation and degeneration by downregulating toll-like receptor 4 . Oncol Rep 40: 793-802, 2018

28. Li Z, Tao Y, Wang X, Jiang P, Li J, Peng M, Zhang X, Chen K, Liu $\mathrm{H}$, Zhen $\mathrm{P}$, et al: Tumor-Secreted exosomal miR-222 promotes tumor progression via regulating P27 expression and Re-localization in pancreatic cancer. Cell Physiol Biochem 51: 610-629, 2018

29. Nie $X$ and Tian H: Correlation between miR-222 and uterine cancer and its prognostic value. Oncol Lett 16: 1722-1726, 2018.

30. Gu J, Wang Y, Wang X, Zhou D, Shao C, Zhou M and He Z: Downregulation of lncRNA GAS5 confers tamoxifen resistance by activating miR-222 in breast cancer. Cancer Lett 434: 1-10, 2018.

31. Lambertini E, Lolli A, Vezzali F, Penolazzi L, Gambari R and Piva R: Correlation between Slug transcription factor and miR-221 in MDA-MB-231 breast cancer cells. BMC Cancer 12: 445, 2012.

32. Brognara E, Fabbri E, Bazzoli E, Montagner G, Ghimenton C, Eccher A, Cantù C, Manicardi A, Bianchi N, Finotti A, et al: Uptake by human glioma cell lines and biological effects of a peptide-nucleic acids targeting miR-221. J Neurooncol 118 19-28, 2014.

33. Penolazzi L, Lambertini E, Bergamin LS, Roncada T, De Bonis P, Cavallo $M$ and Piva R: MicroRNA-221 silencing attenuates the degenerated phenotype of intervertebral disc cells. Aging (Albany NY) 10: 2001-2015, 2018.
34. Loreto C, Musumeci G, Castorina A, Loreto C and Martinez G: Degenerative disc disease of herniated intervertebral discs is associated with extracellular matrix remodeling, vimentin-positive cells and cell death. Ann Anat 193: 156-162, 2011.

35. Wang WJ, Yu XH, Wang C, Yang W, He WS, Zhang SJ, Yan YG and Zhang J: MMPs and ADAMTSs in intervertebral disc degeneration. Clin Chim Acta 448: 238-246, 2015.

36. Li Y, Li K, Mao L, Han X, Zhang K, Zhao C and Zhao J: Cordycepin inhibits LPS-induced inflammatory and matrix degradation in the intervertebral disc. PeerJ 4: e1992, 2016.

37. Wang C, Yu X, Yan Y, Yang W, Zhang S, Xiang Y, Zhang J and Wang W: Tumor necrosis factor- $\alpha$ : A key contributor to intervertebral disc degeneration. Acta Biochim Biophys Sin (Shanghai) 49: 1-13, 2017

38. Yang W, Yu XH, Wang C, He WS, Zhang SJ, Yan YG, Zhang J, Xiang YX and Wang WJ: Interleukin- $1 \beta$ in intervertebral disk degeneration. Clin Chim Acta 450: 262-272, 2015.

39. Wang X, Meng Q, Qiu C, Li P, Qu R, Wang W, Wang Y, Liu L and Zhao Y: Potential therapeutic role of Co-Q10 in alleviating intervertebral disc degeneration and suppressing IL-1 $\beta$-mediated inflammatory reaction in NP cells. Int Immunopharmacol 64: 424-431, 2018.

40. Wang G, Huang K, Dong Y, Chen S, Zhang J, Wang J, Xie Z, Lin X, Fang $X$ and Fan S: Lycorine suppresses endplate-chondrocyte degeneration and prevents intervertebral disc degeneration by inhibiting NF-kB signalling pathway. Cell Physiol Biochem 45: 1252-1269, 2018.

41. Wang J, Pan H, Li X, Zhang K, Li Z, Wang H, Zheng Z and Liu $\mathrm{H}$ : Hypoxia suppresses serum deprivation-induced degradation of the nucleus pulposus cell extracellular matrix through the JNK and NF-kB pathways. J Orthop Res 35: 2059-2066, 2017.

42. Xing F, Zhang W, Wen J, Bai L, Gu H, Li Z, Zhang J, Tao YX and $\mathrm{Xu}$ JT: TLR4/NF-kB signaling activation in plantar tissue and dorsal root ganglion involves in the development of postoperative pain. Mol Pain 14: 1744806918807050, 2018

43. Liao W, He X, Yi Z, Xiang W and Ding Y: Chelidonine suppresses LPS-Induced production of inflammatory mediators through the inhibitory of the TLR4/NF- $\mathrm{B}$ signaling pathway in RAW264.7 macrophages. Biomed Pharmacother 107: 1151-1159, 2018.

44. Sun Z, Jian Y, Fu H and Li B: MiR-532 downregulation of the Wnt/ $\beta$-catenin signaling via targeting Bcl-9 and induced human intervertebral disc nucleus pulposus cells apoptosis. J Pharmacol Sci 138: 263-270, 2018.

45. Gruber HE and Hanley EN Jr: Analysis of aging and degeneration of the human intervertebral disc. Comparison of surgical specimens with normal controls. Spine (Phila Pa 1976) 23: 751-757, 1998.

46. Zhang YH, Zhao CQ, Jiang LS and Dai LY: Lentiviral shRNA silencing of CHOP inhibits apoptosis induced by cyclic stretch in rat annular cells and attenuates disc degeneration in the rats. Apoptosis 16: 594-605, 2011.

47. Zhang W and Liang Z: Comparison between annexin V-FITC/PI and Hoechst33342/PI double stainings in the detection of apoptosis by flow cytometry. Xi Bao Yu Fen Zi Mian Yi Xue Za Zhi 30: 1209-1212, 2014 (In Chinese).

48. Zhang C, Kang C, Wang P, Cao Y, Lv Z, Yu S, Wang G, Zhang A, Jia Z, Han L, et al: MicroRNA-221 and -222 regulate radiation sensitivity by targeting the PTEN pathway. Int J Radiat Oncol Biol Phys 80: 240-248, 2011.

49. Xu Y, Bei Y, Shen S, Zhang J, Lu Y, Xiao J and Li X: MicroRNA-222 promotes the proliferation of pulmonary arterial smooth muscle cells by Targeting P27 and TIMP3. Cell Physiol Biochem 43: 282-292, 2017.

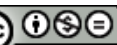

This work is licensed under a Creative Commons Attribution-NonCommercial-NoDerivatives 4.0 International (CC BY-NC-ND 4.0) License. 